\title{
CEREBRAL VEINS AND DURAL SINUS THROMBOSIS: A REVIEW OF 73 CASES
}

Authors: Dragos Catalin Jianu ${ }^{1 *}$, Silviana Nina Jianu², Flavius Dan ${ }^{1}$, Georgiana Munteanu${ }^{1}$, Ligia Petrica $^{3}$

1 "Victor Babes" University of Medicine and Pharmacy, Clinical Emergency County Hospital, Department of Neurology, Timisoara, Romania

${ }^{2}$ Military Emergency Hospital, Department of Ophthalmology, Timisoara, Romania

3 "Victor Babes" University of Medicine and Pharmacy, Clinical Emergency County Hospital, Department of Internal Medicine-Nephrology, Timisoara, Romania

E-mail: dcjianu@yahoo.com

\section{BACKGROUND}

Cerebral veins and dural sinus thrombosis (CVT) generally manifest in various non-specific clinical forms.

\section{AIMS}

To identify CVT causes and risk factors, to describe the demographic, clinical, laboratory, and neuroimaging data, and to evaluate the treatment and outcome.

\section{PATIENTS AND METHODS}

- We analyzed 73 CVT consecutive patients, that were examined at admission (between January 1998 and November 2016), at 90 days, and after twelve months, using the mRS scores.

- The identification of C.V.T, was made in all cases by CT, MRI, MR-A, and, in selected cases, by digital subtraction angiography (DSA).

\section{RESULTS}

Demographic data

mean age: 37.3 years (SD 7.5)

- sex ratio: male/female: $25: 48$ (79.2\% of women being fertile)

Mode of onset

- acute $(<48$ hours): 9 cases $(12.3 \%)$

- $\quad$ sub acute (1-4 weeks): 51 cases $(69.9 \%)$

- chronic (>4 weeks): 13 cases (17.8\%)

\section{Neurological syndromes at onset}

- intracranial hypertension, in 41 patients $(56.2 \%)$

- focal neurological signs (paresis, seizures, and/or aphasia), in 39 patients $(53.4 \%)$

- cavernous sinus thrombosis, in 5 patients $(6.8 \%)$;

- sub acute encephalopathy, with depressed level of consciousness, in 12 cases $(16.4 \%)$

Neuroimaging data

CT (73 cases):

- Direct signs of dural sinuses thrombosis in 12 cases:

a).dense triangle sign (figure 1, figure 6A) in 4 cases,

b).empty delta sign (figure 2) in 13 cases.

- Indirect signs of dural sinuses thrombosis:

a). venous cerebral infarction in 28 cases,

b).cerebral edema in 37 cases,

c) intense contrast enhancement of the falx and tentorium in 8 patients.

- Emissary vein thrombosis- in 5 cases (figure 7)

\section{MRI+/-MRA (73 cases):}

- Direct signs of dural sinuses thrombosis in 59 cases

a). thrombosis of superior sagital sinus (SSS) (figures 3-5) in 47 cases,

b).thrombosis of lateral sinuses (LS) (figures $5,6 \mathrm{~B}, \mathrm{C}$ ) in 30 cases,

c).thrombosis of cavernous sinus in 5 patients,

d).thrombosis of straight sinus in 8 cases,

e).thrombosis of superior petrous sinus in 1 case.

- Indirect signs of dural sinuses thrombosis in 73 cases

a). cerebral edema in 52 cases

b).a venous cerebral infarction (figure 5) in 43 cases

- 19 out of 73 MRI had a normal prior CT.

DSA (5 cases)

- revealed isolated cortical vein occlusion, without sinus occlusion in 5 cases

- prior CT, MRI, and MR-A were normal.

Localization of thrombosis

- one sinus: 17 cases (23.3\%) (5 cavernous sinus, 6 SSS, 6 LS);

- one sinus and cortical veins: 14 cases $(19.2 \%)$;

- two or more sinuses and cortical veins: 37 cases $(50.7 \%)$;

- cortical veins (without sinus occlusion): 5 cases $(6.8 \%)$

\section{Etiology}

a) unknown: 23 cases $(31.5 \%)$

b). known: 50 cases $(68.5 \%)$

- infectious causes: 9 cases (suppurative process of the upper one-half of the face: 5 cases;

otomastoiditis: 4 cases);

- inherited thrombophilia: 25 patients (FV Leiden mutations in 18 cases, prothrombin

(G20210A) mutation in 10 cases, MTHFR C 677T ( 3 patients), protein C deficiency in 2

cases));

pregnancy or puerperium: 18 females

- oral contraceptives: 9 females; etc.

Treatment

All patients received anticoagulant therapy, and peculiar symptomatic and etiologic treatment.

After admission and diagnostic in hospital

The mRS scores of the 73 cases were: level 1-2: 23 cases; level 3-5: 50 cases.

Outcome at 90 days from admission in hospital

- the functional outcome was good, with a mRS score $\leq 2$ in 40 cases, moderate/ severe disability in 25 cases.

- deaths: 8 cases $(10.9 \%)$ :

a). in hospital: 5 patients (massive hemorrhagic cerebral infarct 3 cases, pulmonary embolism 2 cases);

b). after discharge: 3 cases (pulmonary embolism);

- relapses of thrombosis: 7 cases (C.V.T. 3 cases, thrombosis of profound veins of the legs: 4 cases);

seizures after discharge: 6 patients;

\section{Outcome at 12 months from admission in hospital}

- the functional outcome was good, with a mRS score $\leq 2$ in 43 cases $(65.8 \%)$, moderate/severe disability in 22 cases (30.1), the death rate being $10.9 \%$ ( 8 cases).

- CVT severity was found to be associated with presence of rapidly worsening symptoms ( $p=$ $0.001)$, and occlusion of 4 or more sinuses $(p=0.005)$.

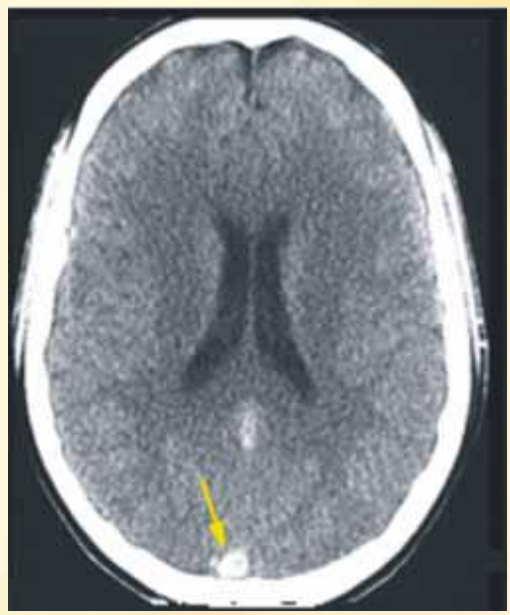

Fig1. Axial Unenhanced CT scan. Dense triangle in a recent S.S.S thrombosis. The thrombus is hyperdense (arrow).

Fig 2. Axial Enhanced CT scan. Empty delta sign. The thrombus is hypodense within the SSS, whose walls are clearly enhanced by the injection (same patient 10 days

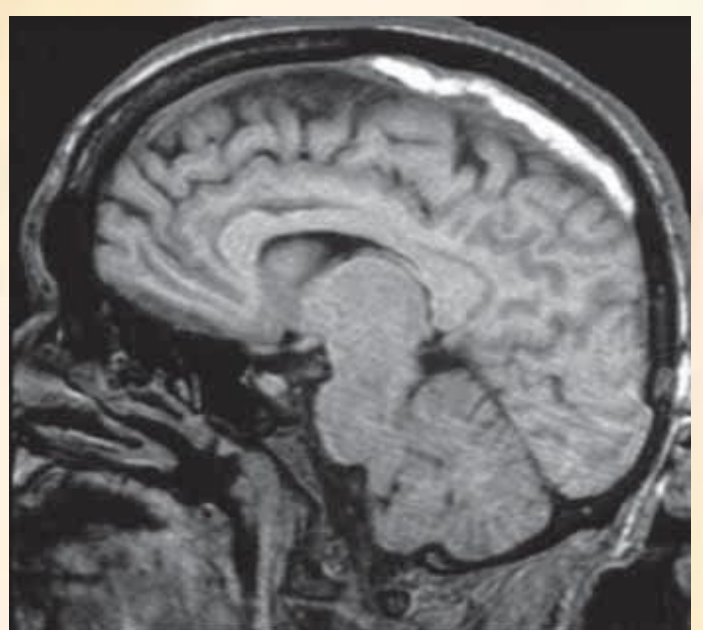

Fig 3. M.R.I., T1-weighted image in the sagittal plane. Hyperintense signal indicating subacute thrombosis of the S.S.S.: (this pattern was found 14 days after the onset of symptoms).

Fig 4. M.R.I., T1-weighted image in the sagitt plane. Hypointense signal indicating chronic thrombosis of the S.S.S.; (same patient, 1 mon
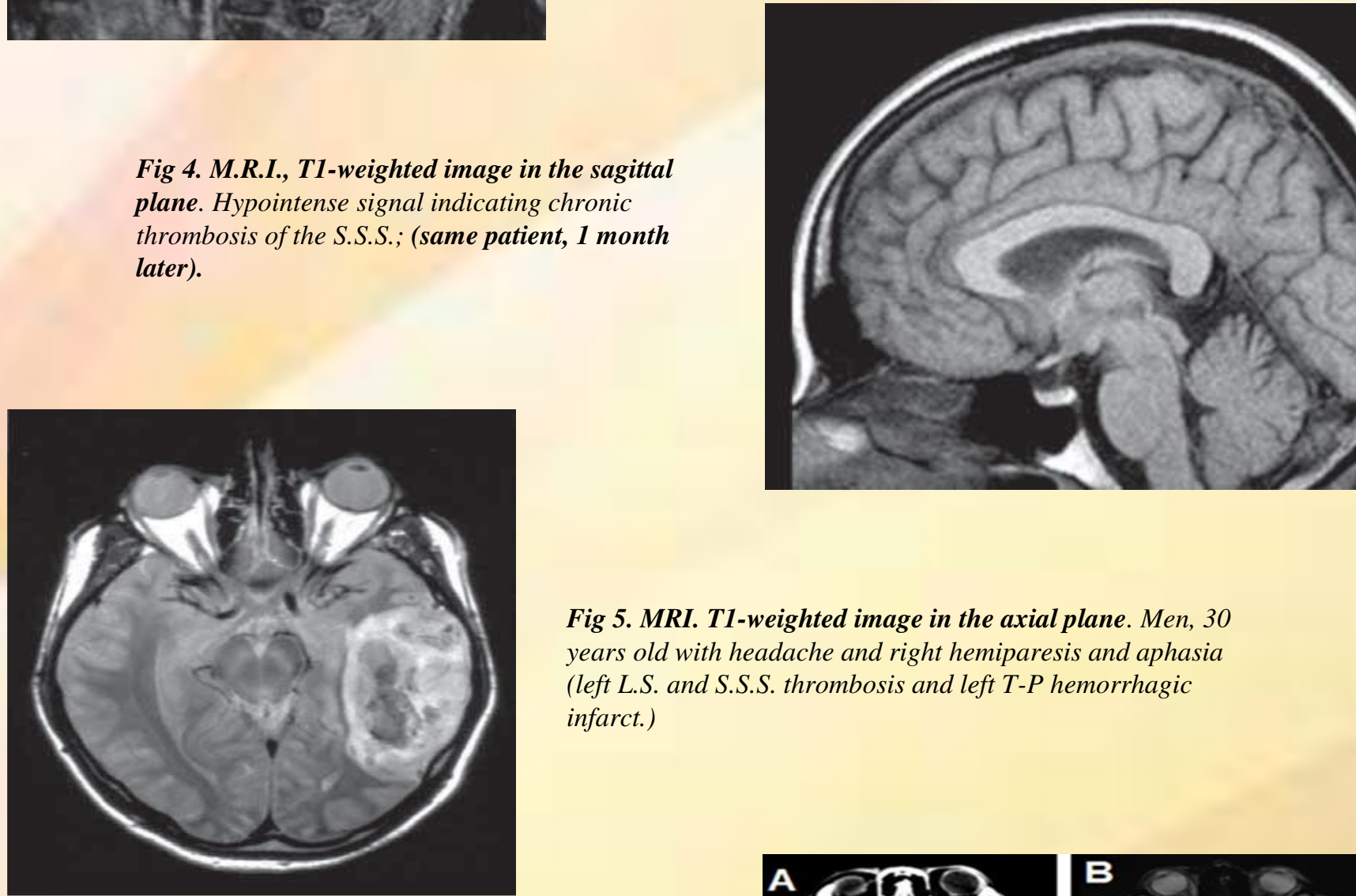

Fig 5. MRI. T1-weighted image in the axial plane. Men, 30 years old with headache and right hemiparesis and aphasia (left $L . S$. and S.S.S. thrombosis and left T-P hemorrhagic infarct.)

Figure 6A. Nonenhanced computed tomography (CT) in the axial plane demonstrating spontaneous hyperdensity of the left (LS) (white arrow).

Figure 6B. Magnetic resonance (MR) T2*SW imaging in the axial plane demonstrating left $L S$ (MR) Thas (Whire ang in the Figure $6 C$. Mastrating left LS hyposignal (white arrow). coronal plane noting absence of $f($ in in the left LS (tho in the of the left $L S$ ) (white arrow).
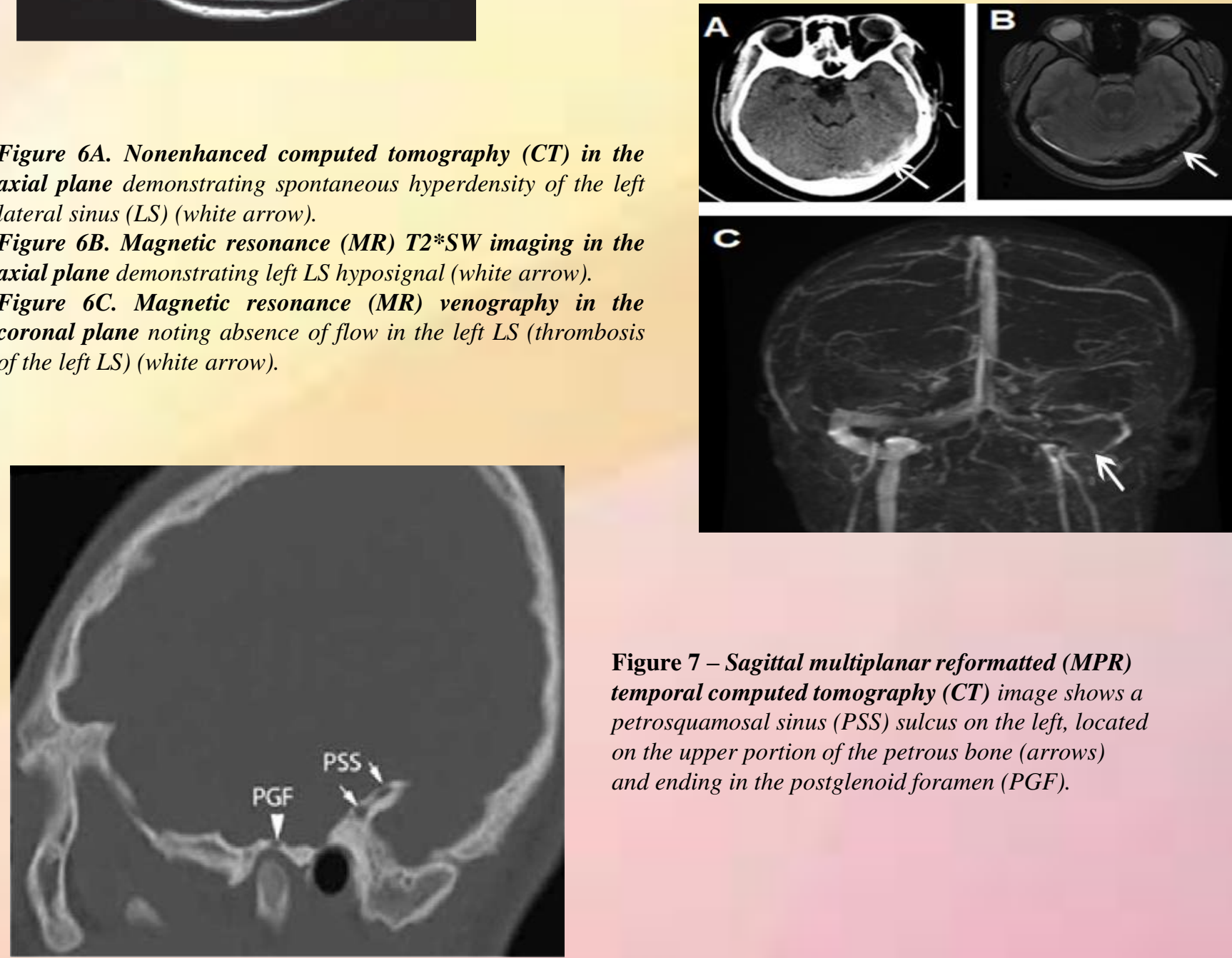

C

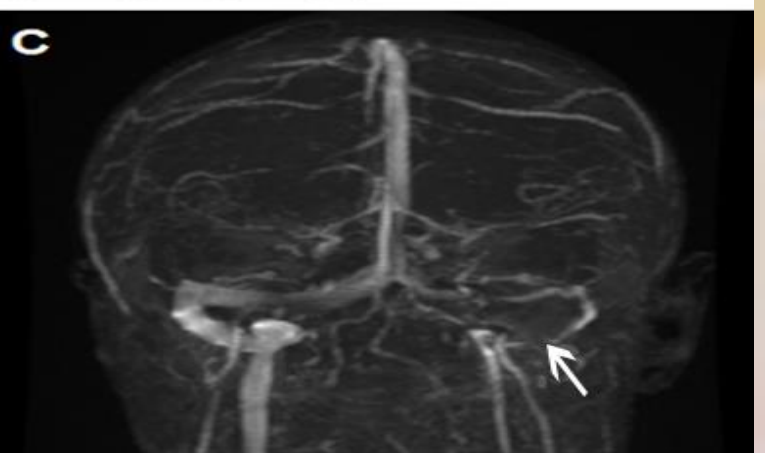

Figure 7 - Sagittal multiplanar reformatted (MPR) Teme temporal computed tomography $(\boldsymbol{C T})$ image shows petrosquamosal sinus (PSS) sulcus on the left, located on the upper portion of the petrous bone (arrows) and ending in the postglenoid foramen (PGF).

\section{CONCLUSIONS:}

- $\quad$ CVT was common in women of fertile age.

- The outcome was favorable if the patients were promptly diagnosed and adequately treated. 